\title{
Indicadores de produção científica na concepção teórica da indexação: uma análise bibliométrica do periódico The Indexer
}

Indicadores de la producción científica en la corriente teórica de la indización: un análisis bibliométrico de la revista The Indexer

Scientific production indicators in the theoretical approach to indexing: a bibliometric analysis of the journal The Indexer

\section{Roberta Dal'Evedove TARTARotti (1), Paula Regina DaL'Evedove (2), Vera Regina Casari Boccato (3), Mariângela Spotti Lopes FuJITA (4)}

(1) roberta_tartarotti@yahoo.com.br $(2,3,4)$ Universidade Estadual Paulista, Av. Hygino Muzzi Filho, 737, Campus Universitário, CEP. 17525900, Marília, SP, Brasil, (2) p.dallevedove@gmail.com (3) vboccato@ufscar.br (4) fujita@marilia.unesp.br

\begin{abstract}
Resumen
Se presenta un análisis de los indicadores de citas de artículos publicados en la revista The Indexer entre 2005 hasta 2012. Se procedió al análisis, preparación, almacenamiento, integración y tratamiento bibliométrico de los datos utilizando Excel y Pajek. Los indicadores bibliométricos de producción científica analizados fueron la distribución de artículos científicos y co-autoria por años; la distribución de artículos publicados por sección; la frecuencia de las palabras clave de los artículos identificados; los actores más productivos; análisis de citas y co-citas; la distribución de citas a plazos; las publicaciones más citadas; y la red de co-citas de los autores más citados, identificando el frente de investigación en el enfoque de la revista.
\end{abstract}

Palabras clave: Indización. Comunicación científica. Indicadores de producción científica. Análisis bibliométrico. Análisis de citas.

\section{Introdução}

No âmbito da Ciência da Informação, a bibliometria, termo cunhado por Paul Otlet como bibliométrie, no Traité de documentation, em 1934, (Fonseca, 1973, p. 5), pode ser definida como o campo científico que objetiva quantificar os processos da informação registrada, envolvendo a análise quantitativa de sua produção, disseminação e uso, no intuito de investigar determinados fenômenos.

Desse modo, o ponto central da bibliometria é "a utilização de métodos quantitativos na busca por uma avaliação objetiva da produção científica" (Araújo, 2006, p.12). Em síntese, trata da medição da comunicação escrita.

Como produtos da bibliometria, as análises bibliométricas não são exclusivas da Biblioteconomia ou da Ciência da Informação: diversas

\begin{abstract}
A bibliometric analysis of the articles published in the journal The Indexer, from 2005 to 2012, is presented. The bibliometric data was analysed, prepared, stored, treated and integrated using Excel and Pajek. Several bibliometric indicators of scientific production were highlighted: distribution of scientific papers and coauthors by year; distribution of articles published by section; frequency of keywords in the identified articles; most productive actors; quotation and co-citation analysis; distribution of citations by fascicles; most cited publications; and co-citation network of the most cited authors, identifying the research front in the journal focus.
\end{abstract}

Keywords: Indexing. Scientific communication. Scientific production indicators. Bibliometric analysis. Citation analysis.

áreas do conhecimento utilizam os estudos de produtividade de autores (Ferreira, 2010).

Estas abordagens pelas quais a ciência pode ser retratada através dos resultados que alcançam são baseadas na prerrogativa de que o objetivo principal da pesquisa científica "é a produção de conhecimento e que a literatura científica é um componente desse conhecimento" (Macias-Chapula, 1998, p.137).

A constituição da bibliometria como uma área na qual incide uma preocupação sobre a medição da informação registrada, é perpassada, na atualidade, por outras subáreas e seus respectivos termos - não consensuais na literatura-, utilizados dependendo de cada contexto em que são aplicados: informetria (direcionada ao estudo dos aspectos quantitativos da informação em qualquer formato e a qualquer grupo social); cientometria (foco nos documentos científicos, 
na ciência e em seus processos de comunicação); webmetria (voltada para o estudo do conteúdo e da estrutura das home-pages na $w e b$; e mais recentemente, patentometria (análise do fluxo da construção do conhecimento baseada na produção de patentes).

De acordo com Vanti (2002, p.153), cada uma dessas abordagens propõe medir a difusão do conhecimento científico e o fluxo da informação sob enfoques diversos. Embora possuam características específicas, ao mesmo tempo convergem na mesma função: medir o fluxo da informação registrada (Zhu et al., 1999, p. 4):

A bibliometria baseia-se na contagem de artigos científicos, citações e patentes. Dependendo da finalidade do estudo bibliométrico, podem ser considerados como dados tanto o texto que compõe a publicação como os elementos presentes em registros sobre publicações extraídos de bases de dados bibliográficas, como nomes de autores, títulos, idioma, palavras-chave e citações.

Considerando-se a relevância dos estudos bibliométricos para a dinâmica da ciência e da tecnologia (C\&T), o objetivo desta investigação é realizar uma análise dos indicadores de citações dos artigos publicados no periódico The Indexer, no período de 2005 a 2012. Por meio da análise das referências bibliográficas de cada artigo, foi possível destacar os autores mais citados no período, bem como identificar a frente de pesquisa no periódico em análise, no intuito de contribuir para a compreensão do fluxo informacional da produção de novos conhecimentos e da dinâmica teórica em torno da temática da indexação neste tipo de publicação científica.

\section{Os indicadores bibliométricos de produção científica}

A bibliometria tem sido utilizada para analisar grandes volumes de publicação científica, dando origem aos denominados indicadores de C\&T, que podem ser definidos como medidas indiretas utilizadas para avaliar elementos intangíveis e complexos, no intuito de compreender a dinâmica do fluxo informacional na ciência e os fatores que determinam a sua evolução que servem como elementos importantes no planejamento, acompanhamento e avaliação de políticas de C\&T.

Desse modo, os indicadores de produção permitem descrever como as informações e os conhecimentos oriundos da atividade científica se relacionam.

São diversos os níveis em que a análise bibliométrica pode ser realizada. Os indicadores de produção científica podem ser classificados em: indicadores de produção (número de publicações por ano, país, instituição, área do conhecimento); indicadores de citação (número de citações recebidas por ano, país, por área do conhecimento, fator de impacto por país e área do conhecimento) e indicadores de ligação (número de coocorrências de autoria, citações cocitações), e palavras (coword).

As citações, na ciência, podem ser definidas como (Foresti, 1989, p.3):

[...] conjunto de uma ou mais referências bibliográficas que, incluídas em uma publicação, evidenciam elos entre indivíduos, instituições e áreas de pesquisa, visto que mostram o relacionamento de uma publicação com outra.

Já a análise de citação investiga (Foresti, 1989, p. 3):

as relações entre os documentos citantes e os documentos citados considerados como unidades de análise, no todo ou em suas diversas partes: autor, título, origem geográfica, ano e idioma de publicação, etc.

No contexto científico, as citações passaram a ter maior relevância principalmente com a criação da base de dados Science Citation In$\operatorname{dex}(\mathrm{SCl})$ pelo Institute for Scientific Information (ISI) e utilização de seus dados sobre citações na avaliação de pesquisadores e instituições (Freitas, 1997).

Um dos pilares da ciência moderna é a divulgação pública das pesquisas e de seus resultados realizadas pelos cientistas ou produtores de informação científica, estimulando a publicação em veículos de divulgação de maior qualidade, visibilidade e melhor avaliados.

Atualmente, a análise de citações é considerada a mais relevante área de estudo da bibliometria, na medida em que identifica e descreve os padrões na produção do conhecimento científico (Araújo, 2006). As cocitações apresentam as relações e frequências dos pares de documentos que são citados conjuntamente por outros documentos, enquanto que (Sinak, 1996, p. 13, 16):

[...] os grupos de cocitações representam a frente de investigação das diferentes áreas do conhecimento, tal como se mostram na literatura citada. Sem dúvida, esses agrupamentos podem representar tanto as redes cognitivas, como as redes sociais entre os investigadores. As redes de cocitações podem traduzir-se em mapas ou nós, onde os pontos denotam documentos e as linhas de união representam as relações de cocitações.

Cabe ressaltar que tanto os periódicos científicos, como as bases de dados, que se constituem as principais fontes para os estudos bi- 
bliométricos, podem ser considerados "amostras" da realidade, pois possuem cobertura limitada, critérios de seleção e propósitos específicos (Martínez, 1994 citado por Baungarten, 2008, p. 162):

Um dos pressupostos da bibliometria é a ideia de ciência como processo de produção (insumoproduto), em que recursos - humanos, capital, equipamentos, materiais (insumos), alimentam condições para a emergência de resultados - artigos de periódicos, livros, patentes, processos (produtos), cuja medição indica a produtividade da ciência.

Entretanto, Baungarten (2008, p. 162) ressalta que os indicadores bibliométricos tradicionais, de caráter quantitativo, foram desenvolvidos para avaliar/medir a ciência nos países centrais e se ajustam à situação desses países e, portanto, sua aplicação para os países periféricos, como o Brasil, precisa, necessariamente, passar por processos de validação.

Baungarten (2008, p. 162), baseada em Castro (1986), apresenta alguns problemas das citações, como o efeito que leva a apoiar mais a quem já é bem citado e citações por críticas ao trabalho, entre outros, não necessariamente por concordância do autor citante do autor citado (Baungarten, 2008, p. 162).

Os indicadores bibliométricos partem da visão clássica da ciência de que o produto da ciência reflete-se totalmente nos instrumentos escritos formais dos cientistas, principalmente nos periódicos. Assim, as investigações produzem novos conhecimentos, e estes são tornados conhecidos por meio das publicações, "cuja função é incentivar a produção científica e obter reconhecimento da coletividade, compartilhando resultados" (Velho, 1994 apud Baungarten, 2008, p. 163).

Além disso, Baungarten (2008, p. 163) põe em discussão a utilização dos indicadores bibliométricos como elemento central e norteador da avaliação e formulação de políticas em C\&T, principalmente nos países periféricos.

\section{A concepção teórica da indexação no âmbito do Tratamento Temático da Informação (TTI)}

Em uma perspectiva social da Ciência da Informação, a área de Organização e Representação do Conhecimento dispõe de elementos teórico-práticos necessários no que tange à produção, tratamento e recuperação da informação/conhecimento científicos na sociedade ao propiciar a comunicação entre a produção e seu uso, com destaque para o Tratamento Temático da Informação (TTI).

Segundo Barité (1997, p. 124), o Tratamento Temático da Informação refere-se

à análise, descrição e representação do conteúdo dos documentos, bem como suas inevitáveis interfaces com as teorias e sistemas de armazenamento e recuperação da informação.

Nessa linha de pensamento, considera-se que o ciclo de operações documentais é a base epistemológica da Ciência da Informação, à medida que objetiva mediar a informação socializada para o indivíduo, produzindo novos conhecimentos. Enquanto os autores/cientistas são considerados os produtores neste ciclo, por sua vez, os consumidores de informação são os usuários/cientistas que se utilizam da informação para produzir novos conhecimentos (Guimarães, 2009, p.106):

Observa-se, destarte, que a organização da informação deve ser entendida como um conjunto de procedimentos que incidem sobre um conhecimento socializado (que, por sua vez, é um produto social e tem uma utilidade social e individual), os quais variam em virtude dos contextos em que são produzidos ou os fins a que se destinam, pois é a partir destes que se desenvolvem os parâmetros de organização.

Após o recebimento da informação científica registrada (publicação), destaca-se o Tratamento Temático da Informação como uma das etapas fundamentais - e intermediárias - visando à recuperação da informação/conhecimento pelos usuários/cientistas no contexto acadêmico, o que Guimarães (2003, p. 100) cunhou metaforicamente como uma "ponte informacional".

O processo de Tratamento Temático da Informação viabiliza a efetiva transferência no ciclo, permitindo não apenas a localização física do documento como também (Guinchat e Menou, 1994, p.122):

a utilização das informações que ele contém baseadas nas necessidades dos usuários, no assunto tratado, nos meios da unidade de informação, nos produtos e serviços fornecidos e na relação custo-eficácia.

A indexação é uma das três vertentes teóricas que sustenta a tríade do Tratamento Temático da Informação: a análise documental (analyse documentaire), de origem francesa; a catalogação de assunto (subject catalog), de origem norte-americana e a indexação (indexing), de origem inglesa. A indexação é denominada como (Gil Leiva, 2008, p. 64, tradução nossa):

um processo executado nos objetos suscetíveis de ser representados mediante conceitos e os pedi- 
dos dos usuários para, em última instância, satisfazer necessidades de informação.

Entretanto, independente da corrente teórica, o Tratamento Temático da Informação tem o intuito de (Martinho, 2010, p.160):

fornecer ao usuário a possibilidade de conhecer o conteúdo de um documento antes mesmo de seu acesso físico e a partir disso decidir se tal material é ou não relevante.

Vistas estas considerações acerca da indexação como uma abordagem teórica do Tratamento Temático da Informação, apreende-se que os periódicos científicos contribuem para o fortalecimento da área de indexação da Ciência da Informação, como o periódico The Indexer, detalhado a seguir.

\section{O periódico científico The Indexer}

O periódico The Indexer: the International Journal of Indexing é publicado pela The Society of Indexers, organização sem fins lucrativos fundada em 1957 como um organismo autônomo profissional de indexadores, com o objetivo de promover a abordagem teórica da indexação, a qualidade dos índices e a profissão de indexador (The indexer, 2013). Como uma companhia limitada, é aberta a qualquer pessoa que é ou pretende estar diretamente envolvida com a indexação. Os membros incluem indexadores especializados em mais de uma centena de diferentes assuntos, desde Contabilidade até Zoologia (The Society..., 2013).

Ademais, é o único órgão autônomo profissional para indexadores no Reino Unido e Irlanda, associado com as organizações de indexação na Austrália, Canadá, China, África do Sul e os Estados Unidos da América. Também colabora com o Chartered Institute of Library and Information Professionals (CILIP), uma associação profissional de bibliotecários, arquivistas e documentalistas (The Society..., 2013).

Embora o foco do periódico The Indexer não seja propriamente a indexação em bibliotecas e a maioria de seus artigos seja publicada em inglês, é uma das mais importantes publicações sobre indexação no âmbito internacional.

Conforme informações oriundas do site (The Indexer, 2013), possui periodicidade trimestral, cujos fascículos, ou números, são publicados nos meses de Março, Junho, Setembro e Dezembro, disponíveis tanto em papel (ISSN 0019-4131) como online (ISSN 1756-0632), mediante assinatura.

Os resumos dos artigos são indexados nas seguintes bases de dados: Information Science
Abstracts; BUBL Information Service; Library and Information Science Abstracts; Library, Information Science and Technology Abstracts e Current Awareness Abstracts. Também é indexado pela base de dados Managing Information e parte do conteúdo reproduzido no periódico francês Documentaliste. Por sua vez, os artigos na íntegra estão disponíveis nas seguintes bases de dados da EBSCO: Library, Information Science \& Technology Abstracts with Full Text e Library Literature \& Information Science Full Text.

No site do periódico, esclarece-se que os artigos na íntegra são normalmente sujeitos à revisão por pares (peer review) e que as opiniões expressas nos artigos avaliados não são necessariamente os mesmos da The Society of Indexers ou de qualquer um de seus membros.

Desde a sua fundação em 1958 até 2007 (volumes 1-25), o periódico The Indexer era publicado duas vezes por ano e a periodicidade dos volumes bienal, em um total de quatro volumes por ano. A partir de 2008 , os volumes (ainda compostos por 4 fascículos) passaram a ser anuais. A versão online dos volumes atuais pode ser acessada através do IngentaConnect (1) e os volumes anteriores a dois anos estão disponíveis no próprio site do periódico.

O estudo em torno de um determinado periódico justifica-se por ser este tipo de comunicação formal a mais utilizada pelos pesquisadores na maioria das áreas científicas, inclusive na Ciência da Informação, tanto no cenário nacional como internacional (Freitas, 1997, p. 120). Além disso, os periódicos de maior qualidade são indexados em bases de dados no intuito de garantir maior visibilidade e disseminação do conhecimento científico no âmbito acadêmico.

\section{Procedimentos metodológicos}

A análise dos artigos do periódico The Indexer como corpus de análise delimitou-se no período de 2005 a 2012, totalizando 8 anos. A busca de todos os artigos deste período foi realizada no Portal de Periódicos da Coordenação de Aperfeiçoamento de Pessoal de Nível Superior (Capes). O período de publicação analisado justifica-se pela disponibilidade de acesso online aos artigos na íntegra apenas neste período.

O Portal de Periódicos é uma biblioteca virtual que reúne e disponibiliza às instituições de ensino e pesquisa no Brasil o melhor da produção científica internacional, contando com um acervo de mais de 33 mil títulos com texto completo, 130 bases referenciais, dez bases dedicadas exclusivamente a patentes, além de outros tipos de materiais como livros, enciclopédias, obras 
de referência, normas técnicas, estatísticas e conteúdo audiovisual (Capes, 2013).

Em virtude dos registros bibliográficos dos artigos serem estruturados em campos (título, autores, resumo, palavras-chave, filiação dos autores, etc.), foram elaboradas listas para cada um dos campos que permitiriam a análise bibliométrica e a análise de citações e cocitações. Esclarece-se que a identificação de cada publicação partiu da própria indicação do periódico no campo intitulado "tipo de documento".

Cabe ressaltar que a opção pelas variáveis bibliométricas na pesquisa foi definida após um estudo exploratório efetuado em todos os artigos publicados no último número do periódico publicado (v. 30, n. 4 de dez. 2013). Desse modo, o presente estudo foi realizado com o objetivo de analisar as referências bibliográficas de cada artigo e identificar os autores mais citados no período abarcado.

A sequência completa do tratamento automatizado da informação baseado na bibliometria incluiu preparação dos dados, armazenamento e integração por meio da criação de base de dados operacional e tratamento bibliométrico. As listas e matrizes geradas no tratamento bibliométrico foram trabalhadas em planilhas eletrônicas para elaboração de rankings, tabelas e gráficos, obtendo-se alguns indicadores e infor- mações sintéticas de alto valor agregado, permitindo a discussão dos resultados.

Considerando que a elaboração de indicadores de C\&T exige a análise de grande quantidade de dados, no campo da bibliometria foram desenvolvidos alguns softwares capazes de contar de forma automatizada os registros bibliográficos e gerar como resultado listas de frequência e de coocorrência de elementos presentes nestes registros. Assim, os autores mais citados foram levantados por meio do software Excel. De modo a obter uma análise de cocitação adequada e representativa, estabeleceu-se uma matriz de $17 \times 17$ autores, quadrada e simétrica, com autores que receberam pelo menos sete citações cada. Por fim, utilizou-se o software Pajek para mapear e visualizar a frente de pesquisa no periódico em questão, a partir dos autores mais citados e que foram cocitados de forma pareada.

\section{Resultados e discussão}

Entre 2005 e 2012 constata-se 546 publicações no periódico em foco (Tabela I), distribuídas em diversas seções: Artigos científicos (240), Bibliografia (4), Estudo de caso (2), Comentários, Notas e/ou Pontos de vista (97), Crítica literária (146), Obituário (16), Carta (24), Ensaio (1), Processos (5), Entrevistas (1) e Revisão de produtos (10).

\begin{tabular}{|c|c|c|c|c|c|c|c|c|c|c|c|c|}
\hline Fascículos & 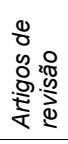 & 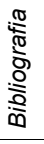 & 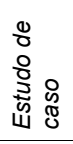 & 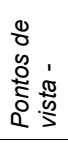 & 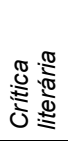 & 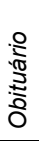 & $\underset{\mathbb{U}}{\mathbb{\pi}}$ & 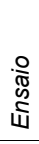 & $\begin{array}{l}0 \\
0 \\
\text { क } \\
0 \\
0 \\
0 \\
0\end{array}$ & 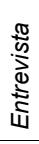 & 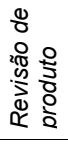 & స్త్ర \\
\hline v. 24 n. $3-2005$ & 13 & 1 & - & 4 & 11 & 3 & - & - & - & - & 1 & 33 \\
\hline v. 24 n. $4-2005$ & 19 & - & - & 5 & 16 & 2 & 1 & - & - & - & 2 & 45 \\
\hline v. 25 n. $1-2006$ & 13 & - & - & 4 & 16 & - & 4 & - & - & - & 1 & 38 \\
\hline v. 25 n. $2-2006$ & 21 & - & - & 12 & 5 & - & 1 & - & - & - & 2 & 41 \\
\hline v. 25 n. $3-2007$ & 13 & - & - & 1 & 12 & - & 1 & - & - & - & - & 27 \\
\hline v. 25 n. $4-2007$ & 14 & - & - & 8 & 8 & 2 & 3 & - & - & - & - & 35 \\
\hline v. 26 n. $1-2008$ & 7 & - & - & 3 & 5 & - & 1 & - & - & - & - & 16 \\
\hline v. 26 n. $2-2008$ & 6 & - & - & 4 & 3 & - & - & - & - & - & 1 & 14 \\
\hline v. 26 n. $3-2008$ & 8 & - & - & 3 & 3 & 2 & 3 & - & - & - & - & 19 \\
\hline v. 26 n. $4-2008$ & 8 & - & - & 3 & 8 & - & - & - & & - & 1 & 20 \\
\hline v. 27 n. $1-2009$ & 8 & - & - & 11 & 7 & 2 & - & - & - & - & 1 & 29 \\
\hline v. 27 n. $2-2009$ & 9 & - & - & 2 & 2 & - & - & 1 & - & - & - & 14 \\
\hline v. 27 n. $3-2009$ & 9 & - & - & 2 & 4 & 2 & 1 & - & 1 & - & - & 19 \\
\hline v. 27 n. $4-2009$ & 5 & - & - & 2 & 4 & - & 1 & - & 2 & - & - & 14 \\
\hline v. 28 n. $1-2010$ & 8 & - & - & 3 & 3 & - & - & - & - & - & 1 & 15 \\
\hline v. 28 n. $2-2010$ & 5 & - & - & 2 & 5 & 1 & 1 & - & - & - & - & 14 \\
\hline
\end{tabular}

Tartarotti, Roberta D.; Dal'Evedove, Paula R.; Boccato, Vera R. C.; Fujita, M. S. L. Indicadores de produção científica na concepção teórica da indexação: uma análise bibliométrica do periódico The Indexer. // Scire. $19: 2$ (jul.-dic. 2013) 45-54. ISSN 1135-3716. 


\begin{tabular}{lllllllllllll}
\hline v. 28 n. 3-2010 & 7 & 1 & 1 & 2 & 6 & 1 & - & - & 1 & - & - & 19 \\
\hline v. 28 n. $4-2010$ & 8 & 1 & - & 5 & 2 & 1 & 3 & - & - & - & - & 20 \\
\hline v. 29 n. $1-2011$ & 5 & - & - & 3 & 4 & - & - & - & - & 1 & 13 \\
\hline v. 29 n. $2-2011$ & 9 & - & - & 4 & 4 & 1 & 2 & - & - & - & - & 20 \\
\hline v. 29 n. 3-2011 & 5 & - & - & 3 & 4 & - & 1 & - & - & - & - & 13 \\
\hline v. 29 n. $4-2011$ & 9 & - & - & 3 & 2 & - & 2 & - & - & - & - & 16 \\
\hline v. 30 n. $1-2012$ & 12 & - & - & 2 & 4 & - & - & - & - & - & - & 18 \\
\hline v. 30 n. $2-2012$ & 12 & - & - & - & 5 & - & - & - & 1 & - & - & 18 \\
\hline v. 30 n. $4-2012$ & 7 & 1 & 1 & 6 & 3 & - & - & - & - & - & - & 18 \\
\hline Total & 240 & 4 & 2 & 97 & 146 & 16 & 24 & 1 & 5 & 1 & 10 & 546 \\
\hline
\end{tabular}

Tabela I. Distribuição dos artigos publicados por seção

\begin{tabular}{|c|c|c|c|c|c|c|c|c|c|c|}
\hline \multirow{2}{*}{$\begin{array}{l}\text { Firmas } \\
\text { Fascículos }\end{array}$} & \multicolumn{2}{|c|}{ Autoria simples } & \multicolumn{2}{|c|}{ Autoria dupla } & \multicolumn{2}{|c|}{ Autoria tripla } & \multicolumn{2}{|c|}{ Autoria n-upla } & \multicolumn{2}{|c|}{ Total de artigos } \\
\hline & $\mathrm{Fi}$ & $\%$ & $\mathrm{Fi}$ & $\%$ & $\mathrm{Fi}$ & $\%$ & $\mathrm{Fi}$ & $\%$ & $\mathrm{Fi}$ & $\%$ \\
\hline v. 24 n. $3-2005$ & 11 & $4,6 \%$ & - & - & 1 & $0,4 \%$ & 1 & $0,4 \%$ & 13 & $5,4 \%$ \\
\hline v. 24 n. 4 - 2005 & 15 & $6,3 \%$ & 1 & $0,4 \%$ & 2 & $0,8 \%$ & 1 & $0,4 \%$ & 19 & $7,9 \%$ \\
\hline v. 25 n. 1 - 2006 & 11 & $4,6 \%$ & 2 & $0,8 \%$ & - & - & - & - & 13 & $5,4 \%$ \\
\hline v. 25 n. 2 - 2006 & 20 & $8,3 \%$ & - & - & - & - & 1 & $0,4 \%$ & 21 & $8,8 \%$ \\
\hline v. 25 n. 3 - 2007 & 11 & $4,6 \%$ & 2 & $0,8 \%$ & - & - & - & - & 13 & $5,4 \%$ \\
\hline v. 25 n. 4 - 2007 & 14 & $5,8 \%$ & - & - & - & - & - & - & 14 & $5,8 \%$ \\
\hline v. 26 n. 1 - 2008 & 7 & $2,9 \%$ & - & - & - & - & - & - & 7 & $2,9 \%$ \\
\hline v. 26 n. 2 - 2008 & 6 & $2,5 \%$ & - & - & - & - & - & - & 6 & $2,5 \%$ \\
\hline v. 26 n. 3 - 2008 & 8 & $3,3 \%$ & - & - & - & - & - & - & 8 & $3,3 \%$ \\
\hline v. 26 n. 4 - 2008 & 7 & $2,9 \%$ & 1 & $0,4 \%$ & - & - & - & - & 8 & $3,3 \%$ \\
\hline v. 27 n. 1 - 2009 & 8 & $3,3 \%$ & - & - & - & - & - & - & 8 & $3,3 \%$ \\
\hline v. 27 n. $2-2009$ & 9 & $3,8 \%$ & - & - & - & - & - & - & 9 & $3,8 \%$ \\
\hline v. 27 n. 3 - 2009 & 6 & $2,5 \%$ & 3 & $1,3 \%$ & - & - & - & - & 9 & $3,8 \%$ \\
\hline v. 27 n. 4 - 2009 & 5 & $2,1 \%$ & - & - & - & - & - & - & 5 & $2,1 \%$ \\
\hline v. 28 n. 1 - 2010 & 8 & $3,3 \%$ & - & - & - & - & - & - & 8 & $3,3 \%$ \\
\hline v. 28 n. 2 - 2010 & 3 & $1,3 \%$ & 2 & $0,8 \%$ & - & - & - & - & 5 & $2,1 \%$ \\
\hline v. 28 n. 3 - 2010 & 7 & $2,9 \%$ & - & - & - & - & - & - & 7 & $2,9 \%$ \\
\hline v. 28 n. 4 - 2010 & 7 & $2,9 \%$ & 1 & $0,4 \%$ & - & - & - & - & 8 & $3,3 \%$ \\
\hline v. 29 n. 1 - 2011 & 4 & $1,7 \%$ & 1 & $0,4 \%$ & - & - & - & - & 5 & $2,1 \%$ \\
\hline v. 29 n. 2 - 2011 & 8 & $3,3 \%$ & 1 & $0,4 \%$ & - & - & - & - & 9 & $3,8 \%$ \\
\hline v. 29 n. 3 - 2011 & 4 & $1,7 \%$ & - & - & 1 & $0,4 \%$ & - & - & 5 & $2,1 \%$ \\
\hline v. 29 n. 4 - 2011 & 8 & $3,3 \%$ & 1 & $0,4 \%$ & - & - & - & - & 9 & $3,8 \%$ \\
\hline v. 30 n. 1 - 2012 & 9 & $3,8 \%$ & 3 & $1,3 \%$ & - & - & - & - & 12 & $5,0 \%$ \\
\hline v. 30 n. 2 - 2012 & 11 & $4,6 \%$ & 1 & $0,4 \%$ & - & - & - & - & 12 & $5,0 \%$ \\
\hline v. 30 n. 4 - 2012 & 7 & $2,9 \%$ & - & - & - & & - & - & 7 & $2,9 \%$ \\
\hline Total de Artigos & 214 & - & 19 & - & 4 & - & 3 & - & 240 & - \\
\hline PORCENTUAL & - & $89,4 \%$ & - & $7,8 \%$ & - & $1,6 \%$ & - & $1,2 \%$ & - & $100 \%$ \\
\hline
\end{tabular}

Tabela II. Distribuição dos artigos científicos por coautoria e ano (Fi = Frequência)

Tartarotti, Roberta D.; Dal'Evedove, Paula R.; Boccato, Vera R. C.; Fujita, M. S. L. Indicadores de produção científica na concepção teórica da indexação: uma análise bibliométrica do periódico The Indexer. // Scire. 19:2 (jul.-dic. 2013) 45-54. ISSN 1135-3716. 
Em um total de 240 artigos apresentados por 195 atores, 214 são oriundos de publicação individual (Tabela II), que correspondem a $89 \%$ das publicações no período abarcado, com maior frequência no ano de 2006 com 31 artigos ou $14 \%$ do total. No período abarcado, tem-se uma média de 30 artigos publicados por ano. Constata-se que apenas $11 \%$ dos artigos publicados no periódico em análise são resultantes de colaboração científica, sendo uma média de 3 artigos publicados por ano fruto da colaboração entre dois ou mais autores.

\begin{tabular}{lc}
\hline Palavras-chave & Frequência \\
\hline Indexing & 124 \\
\hline Indexers & 96 \\
\hline Indexes & 75 \\
\hline Societies, etc. & 24 \\
\hline Information retrieval & 23 \\
\hline Publishers \& Publishing & 23 \\
\hline Subject headings & 21 \\
\hline Documentation & 19 \\
\hline Information organization & 19 \\
\hline Research & 15 \\
\hline
\end{tabular}

Tabela III. Frequência das palavras-chave

\begin{tabular}{lc}
\hline Autores & Frequência \\
\hline Browne, Glenda & 13 \\
\hline Bell, Hazel K. & 10 \\
\hline MacGlashan, Maureen & 9 \\
\hline McMaster, Max & 8 \\
\hline Bosschieter, Pierke & 6 \\
\hline Johncocks, Bill & 5 \\
\hline Russell, Mary & 5 \\
\hline Halliday, Jill & 4 \\
\hline Sassen, Catherine & 4 \\
\hline Alakus, Meral & 3 \\
\hline Coates, Sylvia & 3 \\
\hline Dear, Rosi & 3 \\
\hline Weinberg, Bella Hass & 3 \\
\hline
\end{tabular}

Tabela IV. Atores mais produtivos

Do grupo de 26 artigos publicados em coautoria, verifica-se que a autoria envolvendo dois autores constitui a forma mais frequente de colaboração e representa $73 \%$ dos trabalhos, enquanto a porcentagem de artigos escritos por três ou mais autores foi de $27 \%$. Desse modo, os artigos escritos em colaboração científica representam $11 \%$ do total publicado no período abarcado. Foram identificadas 631 palavras-chave, as quais apareceram 1514 vezes nos 242 artigos publicados, o que equivale a uma média de seis palavras-chave por publicação. A Tabela III apresenta as dez palavras-chave mais frequentes nos artigos analisados. Do total de 195 autores identificados nos 240 artigos, 13 autores publicaram pelo menos três vezes (Tabela IV). Outros 60 autores publicaram duas vezes cada e 152 aparecem com apenas uma publicação.

\begin{tabular}{|c|c|c|c|}
\hline Fascículos & Referências & Artigos & Média \\
\hline v. 24 n. $3-2005$ & 11 & 13 & 1,1 \\
\hline v. 24 n. $4-2005$ & 36 & 19 & 1,8 \\
\hline v. 25 n. $1-2006$ & 51 & 13 & 3,9 \\
\hline v. 25 n. $2-2006$ & 204 & 21 & 9,7 \\
\hline v. 25 n. $3-2007$ & 63 & 13 & 4,8 \\
\hline v. 25 n. $4-2007$ & 109 & 14 & 7,7 \\
\hline v. 26 n. $1-2008$ & 51 & 7 & 7,2 \\
\hline v. 26 n. $2-2008$ & 51 & 6 & 8,5 \\
\hline v. 26 n. $3-2008$ & 36 & 8 & 4,5 \\
\hline v. 26 n. $4-2008$ & 25 & 8 & 3,1 \\
\hline v. 27 n. $1-2009$ & 9 & 8 & 1,1 \\
\hline v. 27 n. $2-2009$ & 81 & 9 & 9 \\
\hline v. 27 n. $3-2009$ & 56 & 9 & 6,2 \\
\hline v. 27 n. $4-2009$ & 31 & 5 & 6,2 \\
\hline v. 28 n. $1-2010$ & 113 & 8 & 14,1 \\
\hline v. 28 n. $2-2010$ & 34 & 5 & 6,8 \\
\hline v. 28 n. $3-2010$ & 28 & 7 & 4 \\
\hline v. 28 n. $4-2010$ & 23 & 8 & 2,8 \\
\hline v. 29 n. $1-2011$ & 26 & 5 & 5,2 \\
\hline v. 29 n. $2-2011$ & 124 & 9 & 13,7 \\
\hline v. 29 n. $3-2011$ & 31 & 5 & 6,2 \\
\hline v. 29 n. $4-2011$ & 94 & 9 & 10,4 \\
\hline v. 30 n. $1-2012$ & 59 & 12 & 4,9 \\
\hline v. 30 n. $2-2012$ & 58 & 12 & 4,8 \\
\hline v. 30 n. $4-2012$ & 100 & 7 & 14,2 \\
\hline Total & 1504 & 240 & 6,3 \\
\hline
\end{tabular}

Tabela V. Distribuição das citações por fascículos

\subsection{Análise de citações e cocitações}

Foram identificadas 1504 referências bibliográficas na elaboração de 240 artigos publicados entre 2005 e 2012 no periódico The Indexer, 
com uma média de 6,3 referências por artigo (Tabela V). A maior frequência de citações ocorreu no volume 25, número 2 de 2006, com 204 referências bibliográficas identificadas em 21 publicações, com uma média de 9,7 referências por artigo, seguido pelo volume 29, número 2 de 2011 e pelo volume 28 , número 1 de 2010 , com respectivamente 13,7 e 14,1 referências bibliográficas por artigo.

Do conjunto de referências obtidas, identificaram-se 87 referências mais citadas que representam 242 citações ou $16 \%$ do total das citações entre 2005 a 2012. Desse conjunto de 87 referências, 13 foram as mais citadas e equivalem a $88(36 \%)$ citações. Outras 68 referências receberam duas citações cada e 6 tiveram três, o que totaliza 74 referências com 154 citações ou $64 \%$ do total.

Entre as 87 publicações mais citadas, 46 publicações (53\%) são artigos de periódicos, sendo a metade (23 ou $50 \%$ ) dessas publicações oriundas do próprio The Indexer, o que indica um significativo consumo de informação do próprio periódico pelos autores que nele publicam. Outras 32 publicações são livros ou capítulos de livros (37\%), 5 (6\%) são resultantes de eventos científicos e apenas 4 oriundas de outros tipos documentais, o que representa $4 \%$ dentre as publicações mais citadas. Destaque seja feito para o livro intitulado Indexing books de Nancy C. Mulvany (2005) da Editora University of Chicago Press que recebeu 20 citações.

Em relação às publicações mais citadas pelos autores dos artigos científicos publicados entre 2005 e 2012, são apresentadas (Tabela VI) as publicações e os respectivos autores que receberam quatro ou mais citações, sendo relacionadas por ordem alfabética de sobrenome do autor.

A partir do conjunto de 1504 citações, foram identificados 1023 autores, o que consiste em uma média de 1,4 citações por autor. Deste grupo, 17 autores foram citados pelo menos sete vezes em um total de 215 citações, ou seja, $14 \%$ das citações. O restante dos autores identificados aparecem com 6 ou menos de 6 citações, dos quais 796 dos autores é citada apenas uma vez, o que sugere autores com baixa frequência de citação pelos pesquisadores que publicam no periódico em análise.

Verifica-se na Tabela VI que entre os autores com alta frequência de citação aparecem N. C. Mulvany com 29 citações; $\mathrm{H}$. K. Bell e H. H. Wellisch com 26 citações cada, grupo que corresponde por $38 \%$ do conjunto de citações entre os autores mais citados. Verifica-se, também, que os pesquisadores $\mathrm{N}$. C. Mulvany e $\mathrm{H}$.
H. Wellisch, além do grande número de citações, também são os autores com maior inserção no periódico em questão, pois foram citados em maior número de artigos. Destacase, ainda, H. Kunze que aparece citado em apenas um artigo entre os analisados, porém com alta citação, considerando que foram consumidas 11 publicações distintas do referido autor, conforme segue:

\begin{tabular}{|c|c|c|}
\hline Autor & $\begin{array}{l}N^{\circ} \text { de citações } \\
\text { recebidas }\end{array}$ & $\begin{array}{c}N^{\circ} \text { de artigos em } \\
\text { que foi citado }\end{array}$ \\
\hline Mulvany, N. C. & 29 & 24 \\
\hline Bell, H. K. & 26 & 14 \\
\hline Wellisch, H. H. & 26 & 22 \\
\hline Weinberg, B. H. & 16 & 10 \\
\hline Booth, P. F. & 13 & 11 \\
\hline Saarti, J. & 12 & 3 \\
\hline Hedden, $\mathrm{H}$. & 11 & 9 \\
\hline Kunze, $\mathrm{H}$. & 11 & 1 \\
\hline Browne, G. & 10 & 9 \\
\hline Zhang, Q. & 9 & 4 \\
\hline Jermey, J. & 8 & 3 \\
\hline Knight, G. N. & 8 & 8 \\
\hline Stauber, D. M. & 8 & 7 \\
\hline Hyvönen, E. & 7 & 1 \\
\hline Leise, F. & 7 & 6 \\
\hline Shatford, L. S. & 7 & 6 \\
\hline Wheatley, H. B. & 7 & 5 \\
\hline
\end{tabular}

Tabela VI. Autores mais citados

Apresenta-se, a seguir, a rede de cocitação construída com os 17 autores mais citados, a fim de visualizar a frente de pesquisa no periódico analisado a partir dos autores mais citados (pelo menos sete citações) e que foram cocitados de forma pareada no período de 2005 a 2012 (Figura I). Esclarece-se que a área dos círculos equivale à frequência com que os autores foram citados e a espessura de cada segmento de reta corresponde à intensidade de cocitação entre os pares.

A região mais densa da rede é composta por Mulvany, Wellisch, Bell, Weinberg e Knight, com alta frequência de cocitações, apesar deste último autor não ser contemplado nos primeiros lugares do ranking de citações. Ainda, os autores Mulvany e Bell possuem os maiores números de conexões com os outros autores que 
compõem a rede, sendo cocitados 55 (76\%) e $47(65 \%)$ vezes, respectivamente.

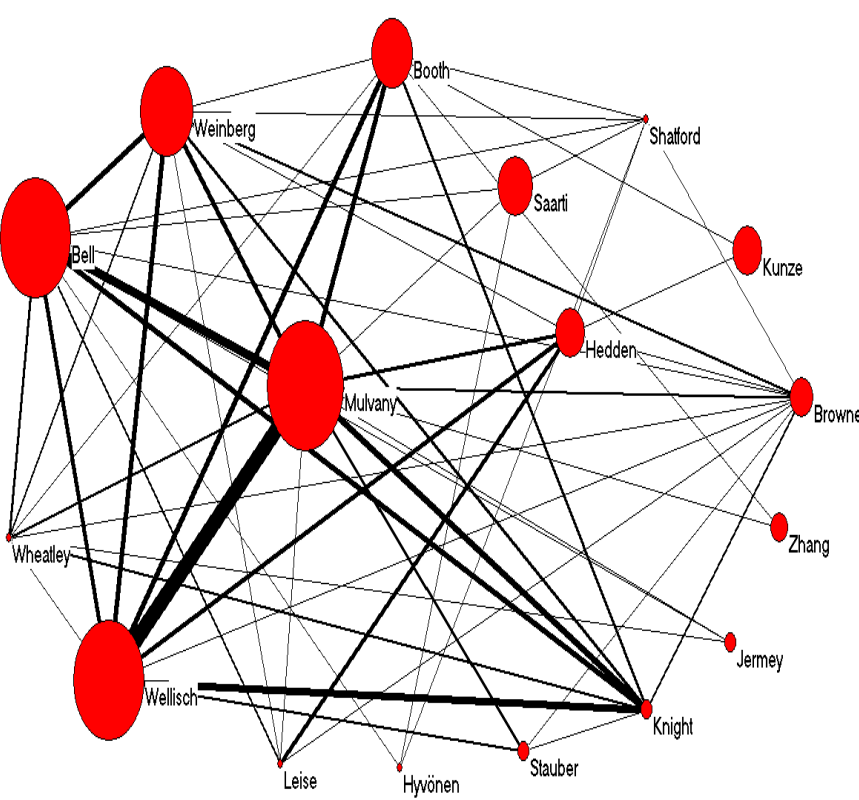

Figura 1. Rede de cocitação dos autores mais citados

Enfatiza-se, ainda, que a densidade da rede (medida de coesão) foi calculada considerando a razão entre as conexões presentes na rede (100) e o número total de conexões possíveis (136), resultando em 0,74 , o que representa $74 \%$ das conexões possíveis foram realizadas na rede, dado que reflete uma alta densidade e pressupõe pesquisas com similaridade ou associação de ideias.

\section{Considerações finais}

Apreende-se que a ciência não é apenas uma descrição isenta da realidade, mas uma prática de construção de conhecimentos e construção de conceitos e de associações inserida e influenciada pelo social, ou seja, uma ciência humana - e que os pesquisadores, produtores do conhecimento científico, são detentores de interesses, valores e éticas próprios.

Verifica-se que os estudos bibliométricos no contexto científico oferecem grande variedade de métodos e medidas como subsídios à avaliação da produtividade acadêmica de indivíduos, grupos, campos científicos, instituições (principalmente as universidades), países, etc. (Borgman; Furner, 2002, p. 30).

Entretanto, a principal restrição à análise de citação é causada pelo fato da citação não medir a qualidade, mas o impacto da publicação. Embora o impacto tenha sido aceito como um importante critério de eficiência dos pesquisadores, para que a avaliação científica tenha credibilidade, não deve ser feita com base em apenas um critério, principalmente porque a publicação não é o único objetivo da pesquisa científica (Velho, 2001).

Cabe ressaltar que, apesar da credibilidade do periódico científico The Indexer sobre a abordagem teórica da indexação no contexto internacional, a falta de padronização nos artigos dificultou as análises bibliométricas, não sendo possível realizar as análises das filiações dos autores e uma análise mais detalhada sobre os mesmos. Além disso, nenhum autor brasileiro foi detectado no período analisado.

Considerando-se a relevância da socialização da informação científica na contemporaneidade e que a produção do conhecimento é indispensável para a produção da ciência, o acesso à informação de qualidade e confiável é imprescindível. A partir desta perspectiva, é preciso ampliar os estudos bibliométricos, contribuindo para traçar um panorama mais amplo sobre o fluxo informacional da área da Organização e Representação do Conhecimento, tanto no contexto internacional como nacional.

\section{Notas}

(1) IngentaConnect é um site que hospeda livros acadêmicos e periódicos científicos a partir de diferentes editoras.

\section{Referencias}

Araújo, C. A. (2006). Bibliometria: evolução história e questões atuais. // Em Questão. Porto Alegre. 12:1 (Jan./Jun. 2006). 1-32. http://periodicos.ufpb.br/ojs/index.php/pbci b/article/view/8023 (2013-03-18).

Baumgarten, M. (2008). Conhecimento e sustentabilidade: políticas de ciência, tecnologia e inovação no Brasil contemporâneo. Porto Alegre: Editora da UFRGS; Sulina, 2008.

Barité, M. (1998). Referenciales teóricos vigentes en el área de tratamiento temático de la información y su expresión metodológica. Porto Alegre: ABEBD, 1998. 7 p. [Relatório técnico do II Encontro de Dirigentes dos cursos superiores de Biblioteconomia dos países do Mercosul, Buenos Aires, nov. 1997].

Borgman, C.; Furner, J. (2002). Scholarly communication and bibliometrics. // Annual Review of Information Science and Technology. New Jersey. 36 (2002) 3-72. http://polaris.gseis.ucla.edu/gleazer/296_readings/arist0 2.pdf (2013-28-03).

The Indexer: the International Journal of Indexing (2013). http://www.theindexer.org (2013-03-15).

Ferreira, A. G. C. (2010). Bibliometria na avaliação de periódicos científicos. // DataGramaZero - Revista de Ciência da Informação. 11:3 (Jun. 2010). http://www.dgz.org.br /jun10/Art_05.htm (2013-28-03).

Fonseca, E. N. (1973). Bibliografia estatística e bibliometria: uma reivindicação de prioridades. // Ciência da Informação. Rio de Janeiro. 2:1 (1973) 5-7. http://revista.ibict. 
br/ciinf/index.php/ciinf/article/viewFile/1625/1234 (201320-03).

Foresti, N. (1989). Estudo da contribuição das revistas brasileiras de Biblioteconomia e Ciência da Informação enquanto fonte de referência para a pesquisa. Dissertação (Mestrado). Brasília: Departamento de Biblioteconomia da Universidade de Brasília, UnB, 1989.

Freitas, M. H. (1997). Oito anos de Transinformação. // Transinformação- Campinas. 9:3 (1997). http://www.bra pci.ufpr.br/documento.php?dd0 $=0000000267 \& d d 1=3891$ 3 (2013-28-03)

Gil Leiva, I. (2008). Manual de indización: teoría y práctica. Gijón: Trea, 2008.

Guimarães, J. A. C. (2003). A análise documentária no âmbito do tratamento temático da informação: elementos históricos e conceituais. // Rodrigues, G. M.; Lopes, I. L. (Org.). Organização e representação do conhecimento na perspectiva da Ciência da Informação. Brasília: Thesaurus, 2003. 100-117.

Guimarães, J. A. C. (2009). Abordagens teóricas de tratamento temático da informação (TTI): catalogação de assunto, indexação e análise documental. // Ibersid. Zaragoza (2009) 105-117.

Guinchat, C.; Menou, M. (1994). Introdução geral às ciências e técnicas da informação e documentação. Brasília: IBICT, 1994.

Macias Chapula, C. A. (1998). O papel da informetria e da cientometria e sua perspectiva nacional e internacional. // Ciência da Informação. 27:2 (1998) 134-140.
Martinho, N. O. (2010). A dimensão teórica e metodológica da catalogação de assunto. Dissertação (Mestrado em Ciência da Informação). Marília: Faculdade de Filosofia e Ciências, Universidade Estadual Paulista "Júlio de Mesquita Filho", 2010.

Portal de Periódicos da Capes (2013). http://www.perio dicos.capes.gov.br (2013-25-03).

Spinak, E. (1996). Dicionário enciclopédico de bibliometria, cientometria e informetria. Venezuela: UNESCO, 1996.

The Society of Indexers. // http://www.indexers.org.uk (201320-03).

Vanti, N. A. P. (2002). Da bibliometria à webometria: uma exploração conceitual dos mecanismos utilizados para medir o registro da informação e a difusão do conhecimento. // Ciência da Informação. Brasília. 31:2 (Maio/Ago. 2002) 152-162. http://www.scielo.br/pdf/ci/v3 1n2/12918.pdf (2013-03-20).

Velho, L. (2001). Cuidado com os rankings científicos. / http://www.prometeu.com.br (2013-03-22).

Zhu, D. et al. (1999). A process for mining science \& technology documents databases, illustrated for the case of "knowledge discovery and data mining". // Ciência da Informação. 28:1 (1999) 7-14. http://dx.doi.org/10.1590/S0 100-19651999000100002 (2013-20-03).

Enviado: 2013-04-06. Segunda versión: 2013-06-06. Aceptado: 2013-09-09. 